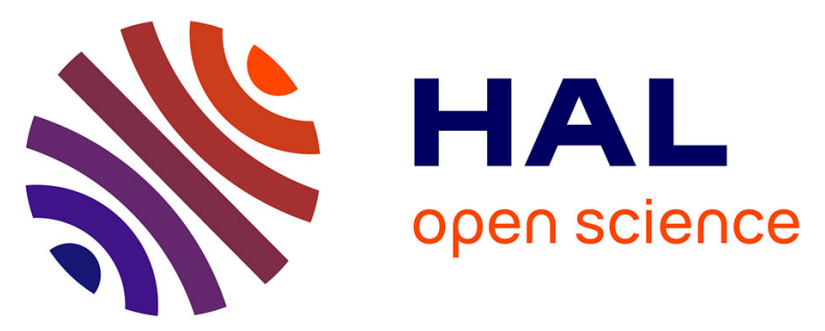

\title{
Confinement of Dyes inside Boron Nitride Nanotubes: Photostable and Shifted Fluorescence down to the Near Infrared
}

\author{
Charlotte Allard, Léonard Schué, Frédéric Fossard, Gaëlle Recher, Rafaella \\ Nascimento, Emmanuel Flahaut, Annick Loiseau, Patrick Desjardins, Richard \\ Martel, Etienne Gaufrès
}

\section{To cite this version:}

Charlotte Allard, Léonard Schué, Frédéric Fossard, Gaëlle Recher, Rafaella Nascimento, et al.. Confinement of Dyes inside Boron Nitride Nanotubes: Photostable and Shifted Fluorescence down to the Near Infrared. Advanced Materials, In press, 10.1002/adma.202001429 . hal-02877816

\section{HAL Id: hal-02877816 https://hal.science/hal-02877816}

Submitted on 22 Jun 2020

HAL is a multi-disciplinary open access archive for the deposit and dissemination of scientific research documents, whether they are published or not. The documents may come from teaching and research institutions in France or abroad, or from public or private research centers.
L'archive ouverte pluridisciplinaire HAL, est destinée au dépôt et à la diffusion de documents scientifiques de niveau recherche, publiés ou non, émanant des établissements d'enseignement et de recherche français ou étrangers, des laboratoires publics ou privés. 


\section{OATAO \\ Open Archive Toulouse Archive Ouverte}

\section{Open Archive Toulouse Archive Ouverte (OATAO)}

OATAO is an open access repository that collects the work of Toulouse researchers and makes it freely available over the web where possible

This is an author's version published in: http://oatao.univ-toulouse.fr/26122

Official URL: https://doi.org/10.1002/adma.202001429

\section{To cite this version:}

Allard, Charlotte and Schué, Léonard and Fossard, Frédéric and Recher, Gaëlle and Nascimento, Rafaella and Flahaut, Emmanuel $\fallingdotseq$ and Loiseau, Annick and Desjardins, Patrick and Martel, Richard and Gaufrès, Etienne Confinement of Dyes inside Boron Nitride Nanotubes: Photostable and Shifted Fluorescence down to the Near Infrared. (2020) Advanced Materials (2001429). 1-10. ISSN 0935-9648

Any correspondence concerning this service should be sent to the repository administrator: tech-oatao@listes-diff.inp-toulouse.fr 


\title{
Confinement of Dyes inside Boron Nitride Nanotubes: Photostable and Shifted Fluorescence down to the Near Infrared
}

\author{
Charlotte Allard, Léonard Schué, Frédéric Fossard, Gaëlle Recher, Rafaella Nascimento, \\ Emmanuel Flahaut, Annick Loiseau, Patrick Desjardins, Richard Martel,** \\ and Etienne Gaufrès*
}

Fluorescence is ubiquitous in life science and used in many fields of research ranging from ecology to medicine. Among the most common fluorogenic compounds, dyes are being exploited in bioimaging for their outstanding optical properties from UV down to the near IR (NIR). However, dye molecules are often toxic to living organisms and photodegradable, which limits the time window for in vivo experiments. Here, it is demonstrated that organic dye molecules are passivated and photostable when they are encapsulated inside a boron nitride nanotube (dyes@BNNT). The results show that the BNNTs drive an aggregation of the encapsulated dyes, which induces a redshifted fluorescence from visible to NIR-II. The fluorescence remains strong and stable, exempt of bleaching and blinking, over a time scale longer than that of free dyes by more than $10^{4}$. This passivation also reduces the toxicity of the dyes and induces exceptional chemical robustness, even in harsh conditions. These properties are highlighted in bioimaging where the dyes@BNNT nanohybrids are used as fluorescent nanoprobes for in vivo monitoring of Daphnia Pulex microorganisms and for diffusion tracking on human hepatoblastoma cells with two-photon imaging. displays. Although they have become ubiquitous in our lives, organic dyes are inherently photodegradable and reactive in physiological conditions. ${ }^{[1]}$ Known since the nineteenth century, ${ }^{[2]}$ the dyes' instabilities stem in part from different photoactivated physical and chemical processes occurring during the lifetime of the excited-state, which include dark state formation by intersystem crossing, ${ }^{[3,4]}$ molecular conformation change, ${ }^{[5]}$ and light-induced charging and triggering of temporary perturbations (blinking) due to random excursions between bright and dark states. ${ }^{[6-8]}$ More significantly, reactive oxidizing species (ROS) placed in contact with the dye induce irreversible photoluminescence (PL) extinction, known as photobleaching or fading. ${ }^{[9,10]}$ These processes drastically reduce the time window to perform experiments and hence limit bioimaging applications and in vivo monitoring in various conditions. For example,

Dye molecules produce intense absorption bands and fluorescence signals over a broad range of wavelengths from the near infrared (NIR) to the ultraviolet (UV). Because of these exceptional properties, they are widely used to color and emit light in various applications, ranging from dyeing fabrics to the green fluorescent protein (GFP) provides a limited number of absorption/emission cycles in the range between $10^{4}$ and $10^{5}$ emitted photons before photobleaching. GFPs remain nevertheless very popular as fluorescence probes albeit their use is limited in typical imaging conditions to only few minutes. ${ }^{[11,12]}$
C. Allard, Prof. P. Desjardins

Département de génie physique

Polytechnique Montréal

Montréal, Québec H3C 3A7, Canada

Dr. L. Schué, Dr. R. Nascimento, Prof. R. Martel

Département de chimie

Université de Montréal

Montréal, Québec H3C 3J7, Canada

E-mail: r.martel@umontreal.ca

Dr. F. Fossard, Dr. A. Loiseau, Dr. E. Gaufrès

Laboratoire d'Etude des Microstructures, ONERA-CNRS, UMR104

Université Paris-Saclay

BP 72, Châtillon 92322, France

E-mail: etienne.gaufres@u-bordeaux.fr

The ORCID identification number(s) for the author(s) of this article can be found under https://doi.org/10.1002/adma.202001429.

\author{
Dr. G. Recher, Dr. E. Gaufrès \\ CNRS \& Institut d'Optique \\ UMR 5298, Talence F-33400, France \\ Dr. G. Recher, Dr. E. Gaufrès \\ LP2N, Laboratoire Photonique Numerique et Nanosciences \\ University of Bordeaux \\ Talence F-33400, France \\ Dr. E. Flahaut \\ CIRIMAT, Université de Toulouse, CNRS, INPT, UPS, UMR CNRS-UPS- \\ INP N5085 \\ Université Toulouse 3 Paul Sabatier \\ Bât. CIRIMAT, 118, route de Narbonne, Toulouse 31062, France
}


Expedients to delay photobleaching have been developed and good examples include the addition of oxygen-blocking molecules, such as anoxia, ${ }^{[13]}$ and a passivation of the dyes against ROS by a polymer, a porous material, or a supramolecular assembly. ${ }^{[14-16]}$ These approaches have had, however, a limited success due to a significant increase in size and a general loss of brightness by the quenchers present in the passivation layers. As an example, inorganic calcium phosphate nanoparticles mixed with dyes (called FloDots) have been developed to prepare photostable fluorescence probes for imaging, ${ }^{[17]}$ but the size constraints $(10-120 \mathrm{~nm})$ do limit the diffusion properties of the probe.

Inspired by these passivation strategies and advances on the encapsulation of organic dyes inside single-walled carbon nanotubes (SWCNTs), ${ }^{[18-21]}$ we explored different hosts to protect dyes while keeping both small (nanometer) sizes and good optical properties (e.g., brightness and stability). Because of efficient energy transfer processes between dyes and SWCNT hosts $\left(E_{\mathrm{g}}<1 \mathrm{eV}\right)$, it became apparent that the dye fluorescence is readily quenched in most, if not all, of the nanohybrids with carbon nanotubes. ${ }^{[22,23]}$ Hence, we turn our attention to the boron nitride nanotube (BNNT), which has a large bandgap $\left(E_{\mathrm{g}} \approx 5.5 \mathrm{eV}\right)^{[24,25]}$ and hence high optical transparency over a wide range of wavelengths. In the context of life science, large diameter BNNTs $(d \approx 50 \mathrm{~nm}$ ) have been studied as cargo for drug delivery ${ }^{[26]}$ and the toxicity in vivo and in vitro of different BNNTs is currently investigated in the field. ${ }^{[27]}$ Here, we present a method to encapsulate dyes inside small-diameter BNNTs (dyes@BNNTs) and explore, using different dyes, their chemical and fluorescence properties for imaging applications. Our experiments on $\alpha$-sexithiophene (6T) and derivatives of 3,6-bis[2,2']bithiophenyl-5-yl-2,5-di-n-oc-tylpyrrolo[3,4c]pyrrole-1,4-dione (DPP) encapsulated inside small-diameter BNNTs $\left(d_{\text {inner }}<3 \mathrm{~nm}\right)$ show a drastic improvement of the photostability compared to free dyes against laser exposure. We observe strong absorption and emission bands from three different e ncapsulated dyes, which a re significantly re dshifted compared to "free" (i.e., not encapsulated) dyes. We also report on an effective passivation of the dyes against the environment. Using various imaging demonstrations with living organisms (Daphnia pulex) and human cells (HuH6), we observe a reduced toxicity compared to free dyes and demonstrate their exceptional photostability. Finally, their use as nanoprobes for multimodal imaging at wavelengths in the visible and NIR is presented along with examples of two-photons imaging and single nanoprobe tracking experiments.

Figure 1 schematizes the main synthesis steps of the dye nanohybrids along with the absorption and fluorescence responses obtained before and after the encapsulation step. The synthesis begins by cutting raw BNNTs (BNNT LLC supplier) using mechanical grinding and ultrasound treatments. The resulting material is then purified in nitric acid and annealed at high temperature in air ${ }^{[28]}$ to suppress photoluminescence background from $\mathrm{BN}$ defects and $\mathrm{B}_{\mathrm{x}} \mathrm{O}_{\mathrm{y}}$ impurities ${ }^{[29]}$ (Figure S1, Supporting information). The last step is a liquid phase encapsulation of a selected dye molecule, for example, DPP2 (Figure 1a), in thus processed BNNTs, followed by a thorough rinsing in dimethylformamide (DMF)/toluene and finally briefly in a piranha solution at room temperature to remove excess of dye molecules. (Warning, piranha solutions are highly corrosive and must be handled with great care while avoiding contact of the solution with residue of organic solvents). The resulting solid residue can be dispersed in a solvent and yields a highly colored solution, such as shown in Figure 1a,c.

The first signature of encapsulation is a clear color change compared to that of free dyes. In Figure 1c, the coloration of the free $6 \mathrm{~T}$ in DMF (P1) is compared to that of a mixture of $6 \mathrm{~T}$ and BNNTs after an encapsulation step in toluene for $24 \mathrm{~h}$ at $25^{\circ} \mathrm{C}(\mathrm{P} 2)$ and $115^{\circ} \mathrm{C}(\mathrm{P} 3)$. No change is observed between P1 and $\mathrm{P} 2$, whereas a clear change from yellow to red is observed for P3. As detailed below, the change in color is driven by temperature and unambiguously indicates that the dyes have filled the BNNTs. Similar spectral changes of the dye resonances are also observed with other rod-like molecules. For example, the derivatives of 3,6-bis[2,2']bithiophenyl-5-yl-2,5di-n-oc-tylpyrrolo[3,4-c]pyrrole-1,4-dione (DPP2 and DPP3) are encapsulated using the same protocol but at $80{ }^{\circ} \mathrm{C}$ (Figure 1c; Section S2.2, Supporting Information). The behavior observed here with BNNTs is fully consistent with previous results from our group on dye encapsulation in SWCNTs. ${ }^{[22]}$ For both types of nanotubes, the filling process is endothermic, due to a heat unbalance from intermolecular interactions between dyes (encapsulated) molecules and between (free) dyes and solvent molecules.

In Figure 1b, we explore more quantitatively the transformation using a comparison of different Dyes@BNNTs (P3, P5, and P7) in DMF with solutions of the same dyes in DMF (P1, P4, P6). The solutions of free dyes, namely 6T (P1), DPP3 (P4), and DPP2 (P6), display absorption (dashed line) and fluorescence (continuous line) spectra characterized by vibronic band progressions that are typical for the dyes in DMF. ${ }^{[30,31]}$ Note that the concentration used for the solutions of free dyes have been adjusted to minimize selfaggregation in solution, which can significantly modify the vibronic progression. ${ }^{32]}$ Surprisingly, the solutions of encapsulated dye molecules (P3-P5-P7) display drastically modified spectra and more complex vibronic structures. DPP3@ BNNT and DPP2@BNNT present remarkable changes, such as new absorption and emissions bands that are significantly redshifted compared to that of free dyes. Interestingly, photoexcitation experiments on P5 and P7 solutions at energies within the absorption bands produce very distinct PL spectra, typically characterized by a broadened vibronic progressions and new emission bands significantly redshifted compared to free dyes. For example, DPP2@BNNT excited at $690 \mathrm{~nm}$ and $800 \mathrm{~nm}$ show emission bands that are clearly shifted at around $900 \mathrm{~nm}$ and 1050-1200 nm, respectively. A similar behavior, although not as drastic, is seen with 6T@BNNT solutions (P3). A more direct comparison of the spectral shifts with that of free dyes is presented in Figure S9, Supporting Information. The unusual redshift could be explained by different phenomena, such as charge transfer, photoinduced electrochromism, structural changes, but these effects have been ruled out by experimental evidences. Charge transfer is impeded considering the good insulating capability of the BNNT sheath from the environment. The wide gap of the BNNTs minimizes also the presence of electronic traps in the proximity of the encapsulated dyes. The selected dyes are rigid 


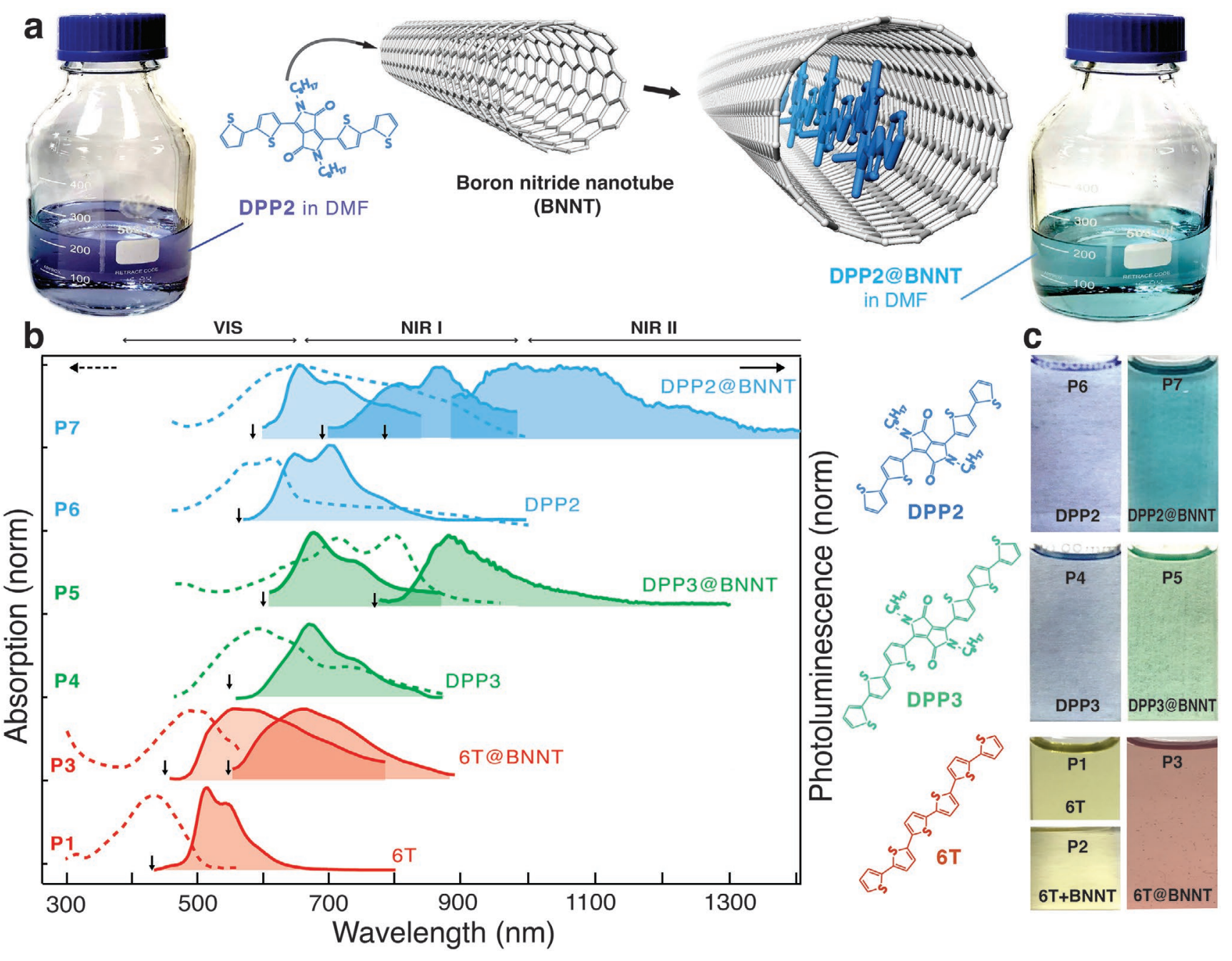

Figure 1. Absorption and fluorescence properties of 6T@BNNTs, DPP2@BNNTs, and DPP3@BNNTs. a) Schematic view of the encapsulation of DPP2 dye in a double-wall BNNT and the resulting color change before and after encapsulation in DMF. b) Absorption (dashed) and fluorescence (continuous) spectra at room temperature of the solutions P1 and P3-P7. The excitation wavelength used for each PL spectrum is indicated by a vertical black arrow. c) Schematics of 6T and DPP-based dyes molecules (left) and pictures of the solutions in DMF before and after a liquid phase (toluene) encapsulation at different temperatures: $25^{\circ} \mathrm{C}$ (P2) and $115{ }^{\circ} \mathrm{C}$ (P3) for $6 \mathrm{~T} ; 80^{\circ} \mathrm{C}$ for DPPs (P5 and P7). P1, P4, and P6 are solutions of free dyes (no BNNT) in DMF.

and planar and they are not prone to conformational changes and photochromism. Furthermore, the mild conditions used for the encapsulation procedure (see Supporting Information) prevent chemical reaction between dyes.

Such drastic changes in optical properties strongly suggest that the inner space of the BNNT has templated the encapsulated dyes into well-defined aggregates. To test this hypothesis, we examine the morphological arrangements of the dyes using high-resolution transmission electron microscopy (HRTEM). Figure 2 shows images of double- and multiwalled BNNTs deposited onto a molybdenum- $\mathrm{SiO}_{2}\left(\mathrm{Mo} / \mathrm{SiO}_{2}\right)$ TEM grid after applying a filling process in solution with 6T molecules. More examples of HRTEM are also shown in Figures S11 and S12, Supporting Information. To determine that the filling material is composed of 6T molecules, preliminary experiments were performed using scanning transmission electron microscopy and energy electron loss spectroscopy (STEM-EELS) recorded at $80 \mathrm{kV}$ to gain information on the chemical composition (core-loss) and the near-band edge response (low-loss) of the filling materials with a spatial resolution of around $2.5 \mathrm{~nm}$ (Figure S10, Supporting Information). The HRTEM image in Figure $2 \mathrm{~b}$ provides distinct responses in the empty and filled regions (open circles 1 and 2, respectively), which are presented in Figure 2c-e. The boron K-edge from the shell structure of the BNNT at $192 \mathrm{eV}$ is measured, as expected, in both regions 1 and 2, but a new contribution is detected only in region 1 at the energy corresponding to the L2,3-edge of the sulfur atoms in a $6 \mathrm{~T}$ molecule. Local energy loss measurements in regions 1 and 2 (Figure 2e) show the expected transitions at around $3 \mathrm{eV}$ (region 1) for the HOMO-LUMO gap of 6T molecules and at $5.5 \mathrm{eV}$ (region 2) for the BNNT bandgap. Consistent to other HRTEM studies on similar oligothiophenes encapsulation inside SWCNTs, ${ }^{[19,20,33,34]}$ the EELS results presented here confirm that the 6Ts are encapsulated inside BNNTs. 

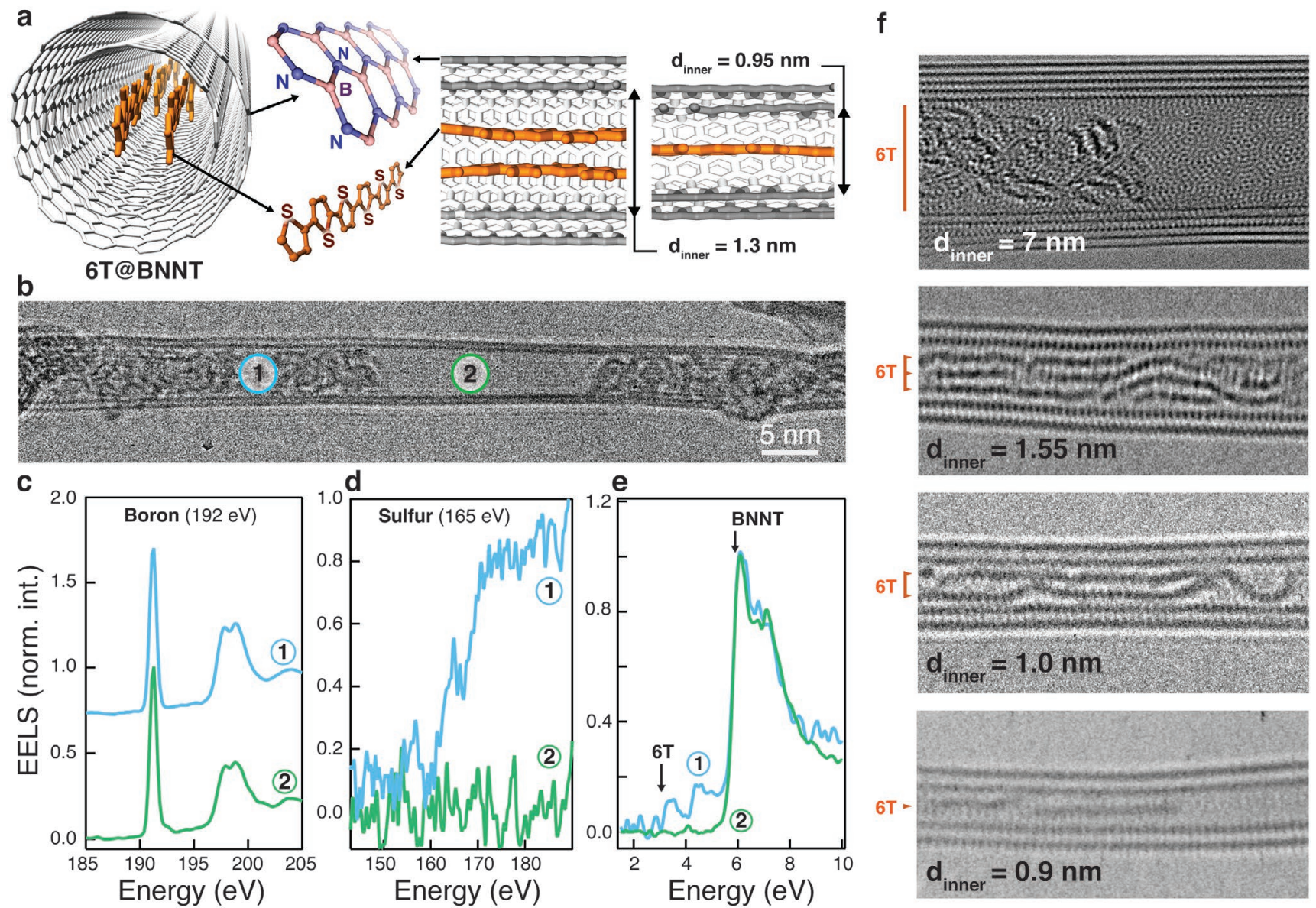

Figure 2. Structural properties of $\alpha$-sexithiophene (6T) encapsulated inside a double-walled boron nitride nanotubes (6T@BNNTs). a) Schematic view of the structure of 6T molecules encapsulated in double-wall BNNTs. b) HRTEM image of a partially filled 6T@BNNT suspended on a molybdenum$\mathrm{SiO}_{2}$ TEM grid. c,d) Chemical analysis of selected areas (1) and (2) in (b) using core-loss STEM-EELS at the boron K-edge (192 eV) and sulfur L2,3-edge $(165 \mathrm{eV})$. e) Measurements of the energy levels in the same two areas by STEM-EELS in the 1-10 eV energy range. f) HRTEM images of 6 T molecules inside BNNTs of different diameters. The HRTEM and STEM-EELS data are recorded at $80 \mathrm{kV}$.

The HRTEM images in Figure 2f show a progressive ordering of the $6 \mathrm{~T}$ dyes, from disordered assemblies to well-aligned individual molecules, for inner diameters $\left(d_{\text {inner }}\right)$ from $7 \mathrm{~nm}$ down to $0.9 \mathrm{~nm}$, respectively. A key result for this study is the presence of single and double aggregates of $6 \mathrm{~T}$ molecules inside BNNTs having the smallest $d_{\text {inner }}$. These aggregates are nicely resolved in Figure $2 \mathrm{f}$ as short sticks (pointing orange arrows) located inside BNNTs of $d_{\text {inner }}=0.90,1.00$, and $1.55 \mathrm{~nm}$ for single, double, and triple aggregates, respectively. Note that the double aggregate is schematically shown in Figure 2a. It is clear from the TEM experiments that high confinement (i.e., low $\left.d_{\text {inner }}\right)$ induces molecular ordering and alignment along the nanotube axis. A cavity of $d_{\text {inner }}=4 \mathrm{~nm}$ is, for instance, much larger (about twice) than the length of the molecule and provides higher degrees of freedom to accommodate various assemblies, whereas $d_{\text {inner }}=0.9 \mathrm{~nm}$ (the smallest diameter observed in our samples) provides just enough space to fit one molecule in a head-to-tail type of stacking.

The assembly of elongated dyes inside BNNTs into aligned and structured aggregates is consistent with what was observed previously with SWCNTs..$^{[1-22,33,35]}$ However, the distribution of the inner dimeters in our BNNT samples is much wider than
SWCNTs, which adds complexity to the population distribution of aggregates. Depending on the diameter, both complex or simple alignments between adjacent molecular transition dipole moments are formed, which lead de facto to a mixture of aggregation states in our samples. As seen in the HRTEM images (Figure 2f), the head-to-tail stacking of the encapsulated dyes in small-diameter BNNTs coexists in solution with face-to-face arrangements in larger diameter BNNTs, giving in some cases double and triple rows of molecules. The former morphology corresponds to the emblematic J-aggregation state, while the latter is consistent with a $\mathrm{H}$-aggregate, each promoting specific intermolecular interactions. ${ }^{[36,37]}$

Considering the diversity of morphologies in our samples, one should therefore expect specific optical signatures associated to possible H-like, J-like, and HJ-states, as well as other photophysical phenomena such as Davidov splitting, excimers and energy transfers. ${ }^{[32,36,37]}$ By shifting the excitation wavelengths well below the gap of free dyes (arrows, Figure 1), we clearly see for instance new vibronic bands that are strongly shifted toward the NIR. This reflects subpopulations of aggregation states that are consistent with J- or HJ-like bright emitters in small-diameter BNNTs. ${ }^{[32,38,39]}$ Such J-aggregation 

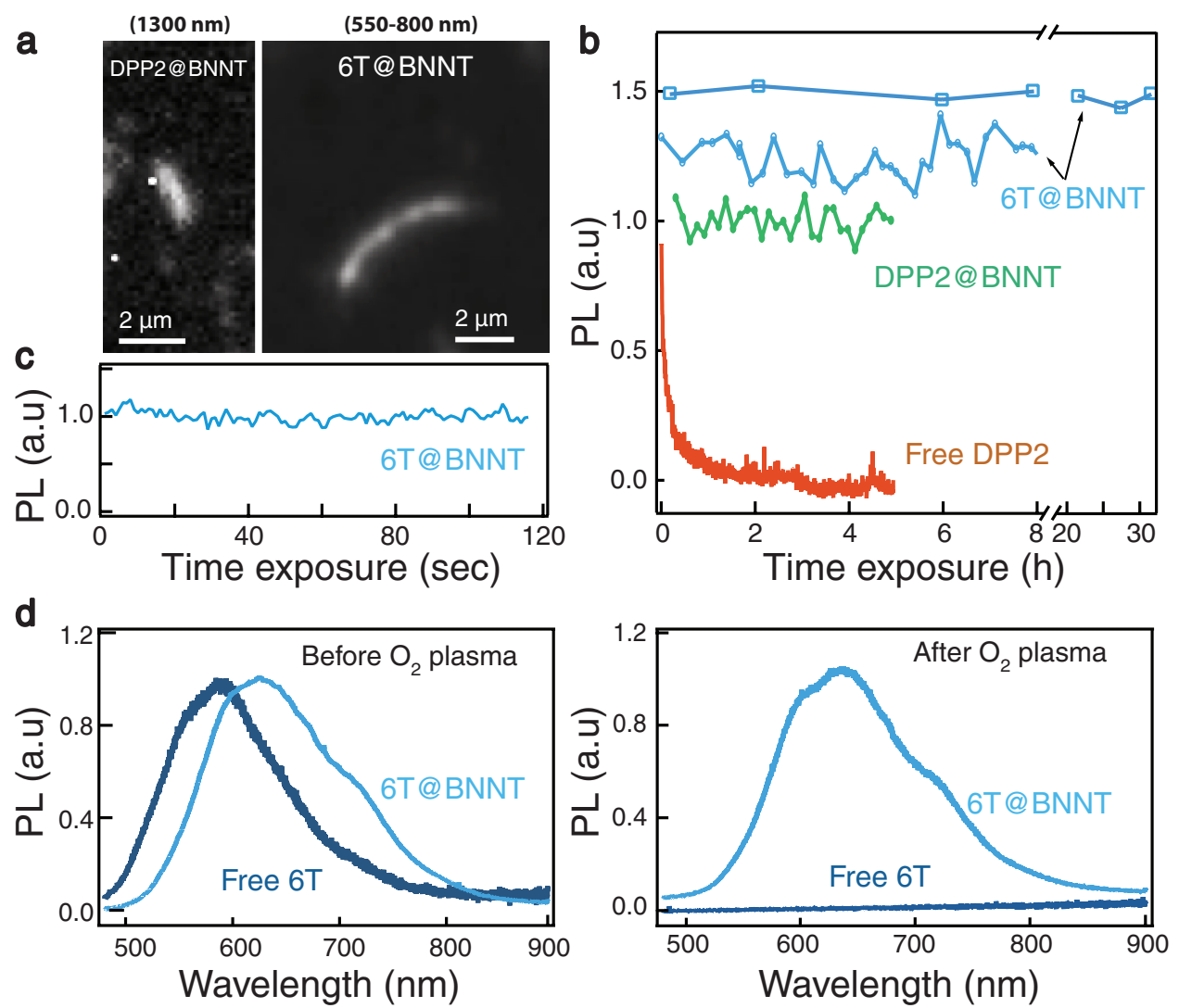

Figure 3. Photostability and chemical resistance of 6T@BNNTs. a) Left panel: Fluorescence image recorded at $1300 \mathrm{~nm}$ (4 nm of spectral interval) from a DPP2@BNNT deposited on a $\mathrm{Si} / \mathrm{SiO}_{2}$ substrate $\left(\lambda_{\mathrm{ex}}=800 \mathrm{~nm}\right)$. The acquisition time is $10 \mathrm{~s}$ at a fluence of $1.0 \mu \mathrm{W} \mu \mathrm{m}^{-2}$. Right panel: Integrated luminescence imaging of a typical microbundle of a 6T@BNNT deposited on a $\mathrm{Si} / \mathrm{SiO}_{2}$ substrate $\left(\lambda_{\mathrm{ex}}=532 \mathrm{~nm}\right)$. The time acquisition is $0.2 \mathrm{~s}$ and the laser fluence is $1.2 \mu \mathrm{W} \mu \mathrm{m}^{-2}$. b) Time evolution of the integrated PL of isolated bundles of 6T@BNNTs (blue) and DPP2@BNNTs (green) on a Si/ $\mathrm{SiO}_{2}$ surface, compared to free DPP2 (red) photoexcited under a fluence of $1.2 \mu \mathrm{W} \mu \mathrm{m}^{-2}$ at $\lambda_{\text {ex }}=532 \mathrm{~nm}$ (long pass filter at $533 \mathrm{~nm}$ ). c) PL intensity recorded at time intervals of $0.5 \mathrm{~s}$. d) PL spectra $\left(\lambda_{\mathrm{ex}}=473 \mathrm{~nm}\right)$ of free 6T and 6T@BNNT deposited on a substrate recorded before (left) and after (right) a $100 \mathrm{~W}$ treatment to oxygen plasma for $10 \mathrm{~min}$.

fingerprints with large redshifts have been previously observed by Sun et al. in self-assembled nitroazo dyes or cyanine dyes and applied in demonstrations of in vivo bioimaging in the NIR II. ${ }^{[40,41]}$ The case of P7 is of particular interest because the emission is redshifted by more than $600 \mathrm{~nm}$, which is surprising for dyes that normally fluoresce in the visible. Understanding the bands and vibronic structures in such a complex mixture of aggregates is obviously not straight forward and clearly outside the scope of this work. Ongoing work explores approaches to disentangle each population of aggregates using for instance sorted BNNTs by inner diameters.

Using $\mathrm{CdSe} / \mathrm{ZnS}$ quantum dots as quantum yield (QY) internal reference, ${ }^{[42,43]}$ we estimated a lower bond value between $8 \%$ and 16\% for 6T@ BNNTs on $\mathrm{Si} / \mathrm{SiO}_{2}$ (see details in Figure S13, Supporting Information). The QY value is significantly higher than $0.5 \%$, which is a typical for $6 \mathrm{~T} \mathrm{films,}{ }^{[44]}$ and it is close to the values measured on individual $6 \mathrm{~T}$ in solution or assemble as J-aggregates. ${ }^{[45]}$

The passivation of the dyes with the crystalline dielectric sheath of the BNNT walls is tested next using stability measurements against photobleaching and chemical reactions. To do so, we compared in Figure 3 the PL properties of free dyes with that of individual or small bundles of 6T@BNNTs deposited on an inert $\mathrm{Si} / \mathrm{SiO}_{2}$ substrate in ambient conditions. A typical AFM image is shown in Figure S2, Supporting Information. Note that an oxide thickness of $\approx 300 \mathrm{~nm}$ was chosen to avoid destructive optical interference with the substrate. To gain statistics, a global hyperspectral imager (RIMA, Photon etc.) was used to photoexcite uniformly a large area of the sample $\left(200 \times 200 \mu \mathrm{m}^{2}\right)$ with continuous laser light ${ }^{[46]}$ at $\lambda_{\mathrm{ex}}=532 \mathrm{~nm}$. This configuration has given us a large statistical ensemble of measurements on individualized bundles of 6T@BNNT as a function of exposure time and fluence conditions. As an example, Figure 2a shows a zoomed view of the PL image of a $5 \mu \mathrm{m}$ long bundle at a laser fluence typical for bioimaging $\left(1.2 \mu \mathrm{W} \mu \mathrm{m}^{-2}\right)$. In Figure $3 \mathrm{~b}$, the PL signal of the bundle shows no noticeable intensity loss with exposure time, even after exposure for more than $8 \mathrm{~h}$ in air. This interesting result indicates a clear resistance against photobleaching, which is also demonstrated with the DPP nanohybrids in Figure 3b. The result contrasts with the fast fading PL signal (in red) of free dyes (not encapsulated) dispersed on the substrate. As expected, the half lifetime of the integrated emission $\left(\tau_{1 / 2}\right)$ of free dyes is only few minutes, which is typical for an organic dye, whereas individual dyes@BNNT bundles produce unaltered PL after more than $30 \mathrm{~h}$. The measurement is only 
a
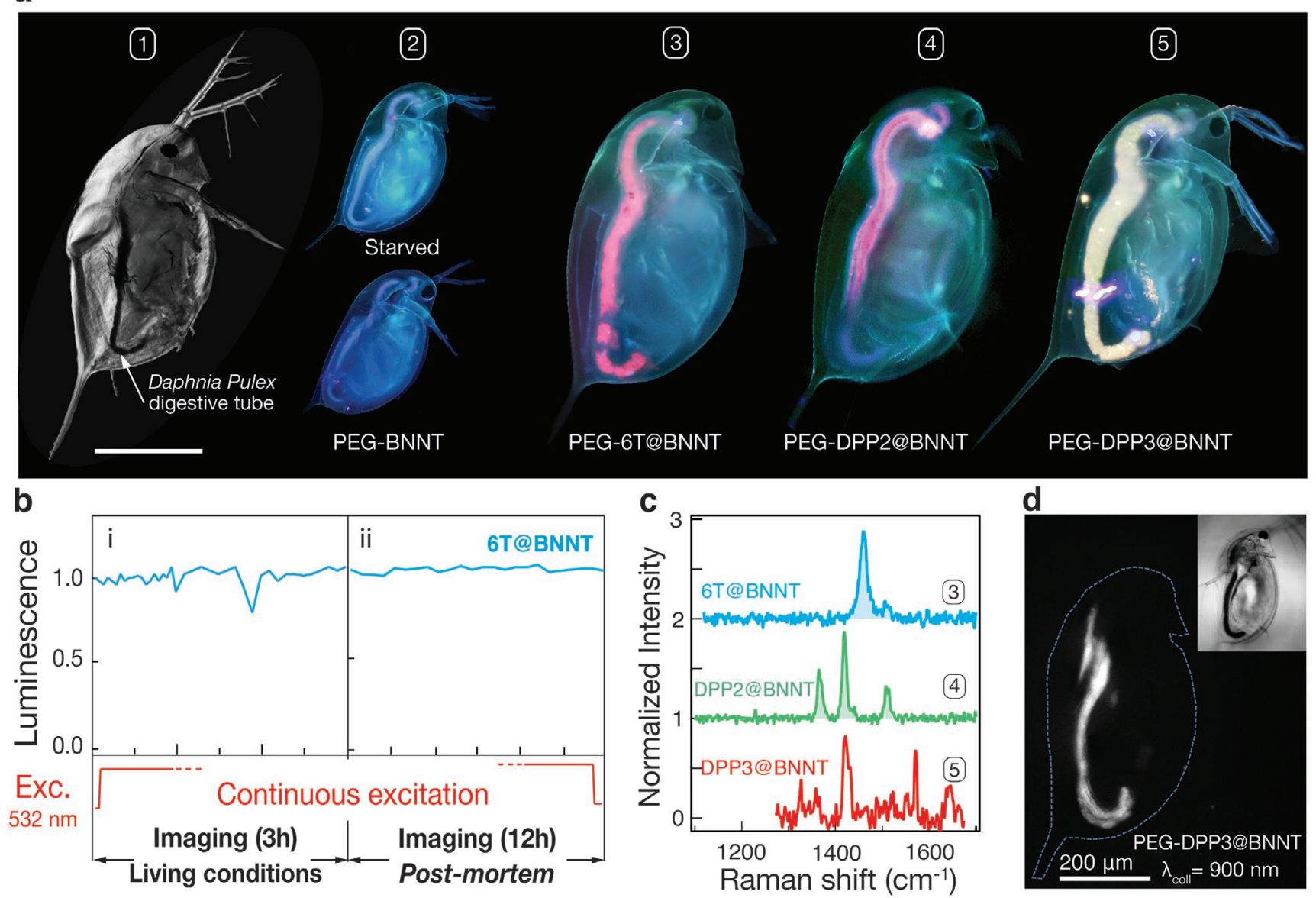

Figure 4. Dyes@BNNTs as hypermodal nanoprobes illustrated for Vis-NIR bioimaging of Daphnia Pulex. a) Z-reconstructed confocal optical image of a Daphnia immobilized in Leica medium (\#1). PL images recorded through a fluorescence microscope mounted with a UBC filter and 10× objective lens of living starved Daphnia (top \#2) and after incubation in a solution of unfilled BNNTs (bottom \#2), 6T@BNNTs (\#3), DPP2@BNNTs (\#4), and DPP3@BNNTs (\#5). b) Luminescence intensity time-lapses of dyes@BNNTs: i) in living conditions, ii) in post-mortem conditions. c) Raman spectra at $\lambda_{\mathrm{ex}}=532 \mathrm{~nm}$ taken in the region of the digestive tube of Daphnia for \#3 and at $\lambda_{\mathrm{ex}}=633 \mathrm{~nm}$ for \#4 and \#5. d) PL image of a living Daphnia collected at a wavelength of $900 \mathrm{~nm}$ using an excitation at $\lambda_{\mathrm{ex}}=532 \mathrm{~nm}$. Inset: Optical image of the Daphnia. The scale bar is $200 \mu \mathrm{m}$ for images \#1, \#3-\#5 and $400 \mu \mathrm{m}$ for \#2.

limited here by our setup to a factor of $1 \times 10^{4}$. Furthermore, PL images taken at the level of a single dye nanohybrid with a frame rate of $0.5 \mathrm{~s}$ indicate no blinking under a fluence of $1.2 \mu \mathrm{W} \mu \mathrm{m}^{-2}$ at $\lambda_{\mathrm{ex}}=532 \mathrm{~nm}$ (Figure $2 \mathrm{c}$ ). These photostability results are impressive considering that this is a lower bound estimate.

The chemical stability of the nanohybrids is tested using individualized6T@BNNT bundles on $\mathrm{Si} / \mathrm{SiO}_{2}$ exposed to an oxygen plasma of $100 \mathrm{~W}$ for $10 \mathrm{~min}$. No significant change in the PL spectral shape and intensity is detected after this treatment, whereas free 6T molecules on $\mathrm{Si} / \mathrm{SiO}_{2}$ have completely vanished (Figure 3d). The enhanced stability of the encapsulated dyes has also been observed upon thermal annealing up to $350{ }^{\circ} \mathrm{C}$ in air and using piranha solutions (not shown). These results demonstrate that the6T@BNNT is far more stable than free 6T dyes. The chemical and physical stability experiments have been repeated on a number of Dyes@BNNT nanohybrids of different lengths deposited on substrates or dispersed in a solution (see, e.g., Figures S5 and S6, Supporting Information). All of the results lead us to the same conclusion, which underlines the tremendous gain in stability inferred by the confinement of the 1D cavity of BNNTs.

The impressive robustness and fading-free properties of the Dyes@BNNT are appealing for bioimaging applications. Figure 4 presents an example of the use of nanohybrids to make optical nanoprobes for imaging living Daphina Pulex microorganisms in water-see bright-field image in Figure 4a\#1. Daphnia (also called water fleas) are common planktonic crustaceans, which find use as chemical sensors in ecotoxicology studies, thanks to their high sensitivity to water quality. ${ }^{[4]}$ To acquire the bioimages (Figure 4a\#2-5), we first solubilized the Dyes@BNNTs in a water-based living medium called FLAMES, ${ }^{[48]}$ using a poly (ethylene) glycol derivative (mPEG-DSPE) (Section S2.9, Supporting Information). We then performed a first incubation test by placing a Daphnia in different FLAMES containing either free 6T, only PEG-BNNTs, or PEG-dyes@BNNTs (see Table S1 and Figure S3, Supporting Information). After an incubation time of only a few minutes, all daphniids exposed to free 6T were found dead. This is consistent with the known toxicity of the dyes to living organisms, 
as evidenced already in the twentieth century by many studies on drugs and contrast agents. ${ }^{[4]}$ On the contrary, all of the Daphnia assays incubated with both PEG-BNNT and PEGdyes@BNNT remain alive and active after incubation times ranging from a few minutes to $36 \mathrm{~h}$ at concentrations between 10 and $50 \mu \mathrm{g} \mathrm{mL} \mathrm{m}^{-1}$ (see video in Figure S4, Supporting Information). This underlines the multiple roles of the BNNT host: i) stabilizing the PL signal from the nanoprobe, ii) acting as a protective barrier against ROS, and iii) reducing dye toxicity to the living organisms. Living daphniids were imaged under a fluorescence microscope equipped with UBG filter and a $10 \times$ objective. D. Pulex incubated with PEG-BNNTs (i.e., no dye, Figure 4a\#2 down) presents similar signal than that observed from non-incubated Daphnia (Figure 4a\#2 top). This natural blue-green signal originates from the autofluorescence of the tissues in FLAMES through the UBG filter, which disappears after death or after a few minutes only of photobleaching. In contrast, Daphniids incubated with PEG-Dyes@BNNTs (Figure 4a\#3-5) exhibit a distinct digestive tube emitting bright signals assigned to the spectral lineshape of each PEG-Dyes@ BNNTs nanohybrids.

In a second set of experiments, we investigated the photostability of the nanoprobes during in vivo PL imaging of $D$. Pulex under continuous light excitation. Daphniids were maintained alive during the first $3 \mathrm{~h}$ of the time-lapse by adding more FLAMES to maintain temperature and compensate water evaporation under the microscope. The PL intensity, recorded every $3 \mathrm{~min}$ at six different points of the digestive tube (Figure $4 \mathrm{a}$ ), remains flat with time, which indicates that bleaching is suppressed in such continuous photoexcitation despite the fact that the nanoprobes are in close contact with the ROS present in the water-based medium of the digestive tube of Daphniids. The brief and temporary drops of signal at $1 \mathrm{~h}$ and $3 \mathrm{~h}$ are attributed to sudden movements of the Daphnia in the exiguous swimming pool under the microscope objective (Figure 4b). Even after death, which is typically caused by a drying of the FLAMES medium, the signal of the nanoprobes remain stable for an additional $12 \mathrm{~h}$ of continuous excitation/ imaging without any noticeable loss of signal (Figure 4b). Interestingly, the Raman spectra in Figure 4c, recorded at the level of the digestive tube of Daphnia \#4 and \#5, show that the specific vibrational fingerprint of the encapsulated 6T (or DPP) in BNNTs remain intact. This signal can be readily extracted and identified from the luminescence signal, thanks to the resonance enhancement of the dyes at $\lambda_{\mathrm{ex}}=532 \mathrm{~nm}$. This experiment demonstrates that the encapsulated dyes are not altered in the digestive tube of the living Daphnia and that they are highly photostable, giving further statistics of the effective passivation by the BNNT hosts.

The capability to probe in situ both the fluorescence and Raman scattering of different nanoprobes over a timescale of many hours is interesting for bioimaging using multimodal probes. Similar to the work on carbon nanotubes based NIR nanoprobes, ${ }^{[50-53]}$ which emit at wavelengths beyond $900 \mathrm{~nm}$, we performed experiments using a DPP3@BNNT incubated in Daphnia. By changing the dyes, we were able to easily extend the PL emission of the nanoprobes (Figure 4d) to the NIR I optical biowindow. ${ }^{[54,55]}$ From this and the results in Figure 1, it is clear that the emission properties of
DPP2@BNNT allows excitation in the NIR I and detection in the NIR II, which is of interest for deep imaging studies on animals such as mice.

To further assess a possible toxicity of the Dyes@BNNT, we performed an additional set of experiments at the scale of individual cells. We incubated $10 \mu \mathrm{g} \mathrm{mL} \mathrm{m}^{-1}$ of a PEG-6T@ BNNT solution for $24 \mathrm{~h}$ with human hepatoblastoma HuH6 cells, which is one of the model cell lines used for toxicology studies. ${ }^{[56,57]}$ After removal of the media containing Dyes@ BNNTs, the cells membranes was revealed using $5 \mu \mathrm{L} \mathrm{mL} \mathrm{m}^{-1}$ of DiA fluorophore for $10 \mathrm{~min}$ (see Supporting Information). Figure 5a,c show fluorescence spectra consistent with DiA (green) and PEG-6T@BNNT (red) separated in the regions 2 and 1 , respectively. In these conditions, we observe that the DiA are fixed, as expected in the cell membranes, while the nanoprobes are internalized by the cells and accumulated in endocytoplasmic compartments. ${ }^{[57]}$ In addition, a positive green Calcein staining assay shows no lethality of the cells in this time scale (Figure S7, Supporting Information). In Figure 5b, we investigated the imaging capability and photostability of the PEG-6T@BNNT using two-photon imaging techniques on HuH6 cells fixed after an incubation of nanoprobes followed by $\mathrm{DiA}$ at a concentration of $10 \mu \mathrm{g} \mathrm{mL} \mathrm{L}^{-1}$ and $5 \mu \mathrm{L} \mathrm{mL} \mathrm{L}^{-1}$, respectively (see Supporting Information). The fluorescence of both the DiA and PEG-6T@BNNT, as a function of the excitation wavelength between 740 and $1020 \mathrm{~nm}$, is shown in Figure 5d. Consistent with the work by Ruthazer et al., ${ }^{[58]}$ the resonance maximum of DiA is measured at $880 \mathrm{~nm}$, which is distinct from the resonance of the PEG-6T@BNNT located at $\approx 740 \mathrm{~nm}$, a position similar to that of functionalized polythiophene. ${ }^{[59]} \mathrm{A}$ wavelength centered at $860 \mathrm{~nm}$ was used in Figure 5a to expose simultaneously DiA and PEG-6T@BNNT in the bleaching test area highlighted (dashed line) with two-photon imaging conditions during $30 \mathrm{~min}(400 \mathrm{~Hz}$, laser output power of $2.55 \mathrm{~W})$. To directly compare exposed and non-exposed cells, Figure $5 \mathrm{~b}$ presents a two-photon hyperspectral fluorescence image taken in a field of view slightly larger than the photobleaching test area. The exposed DiA have been significantly photobleached, while the PEG-6T@BNNTs are still strongly fluorescent without loss of signal. This result highlights the photostable response of the Dyes@BNNT against the stringent two-photon illumination conditions. This result demonstrates long term (day-scale) monitoring without the need of periodic injection of contrast agents.

Finally, the last experiment tracks individual Dyes@BNNT in cellular environment. Because of their 1D architectures, these nanoprobes should have similar diffusion properties than individual SWCNTs, which are advantageous for studies in biological media. ${ }^{[60,61]}$ Using another solution of PEG-6T@ BNNT with an excess of PEG to lower the interaction with the cell membrane $\left(0.5 \mu \mathrm{g} \mathrm{mL} \mathrm{m}^{-1}\right.$ in DMEM), we incubated the nanoprobes for $24 \mathrm{~h}$ followed by rinsing to remove non-interacting nanoprobes with the confluent cell mat (see Supporting Information). Time-lapse luminescence during $30 \mathrm{~min}$ with a time interval $\Delta t$ of $650 \mathrm{~ms}$ between two images is shown in Figure 5e. The superposition of few sequential fluorescence images of a single6T@BNNT nanoprobe, extracted for clarity from the datacube every $\approx 10 \mathrm{~s}$, is displayed in the top panel of Figure 5e. By using a single object tracking algorithm, ${ }^{[62]}$ 


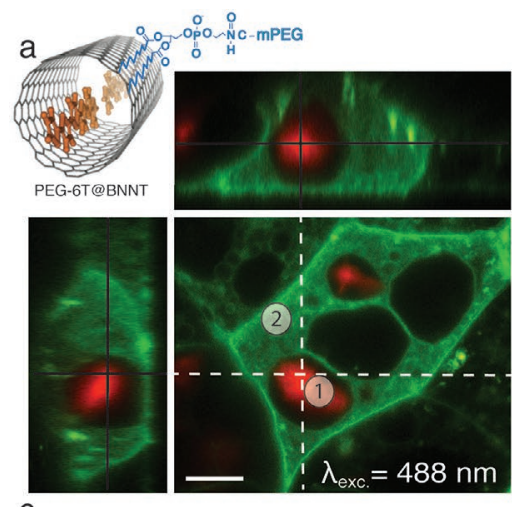

C

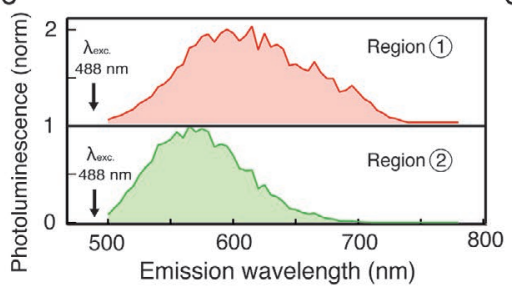

b

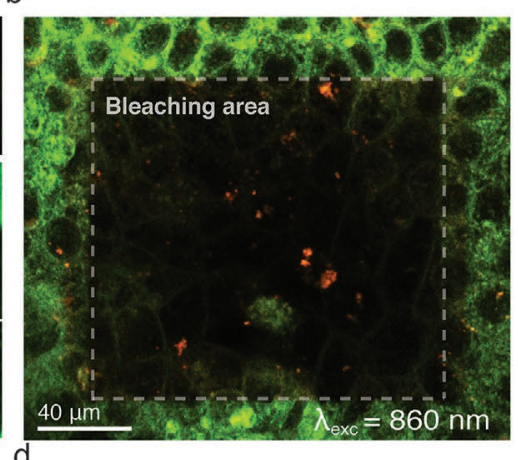
d

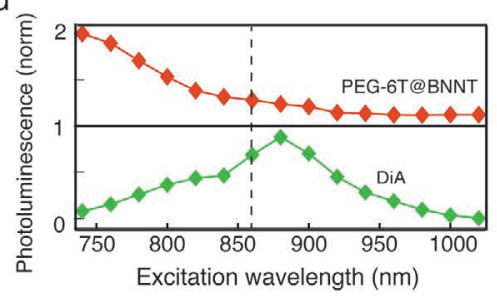

e

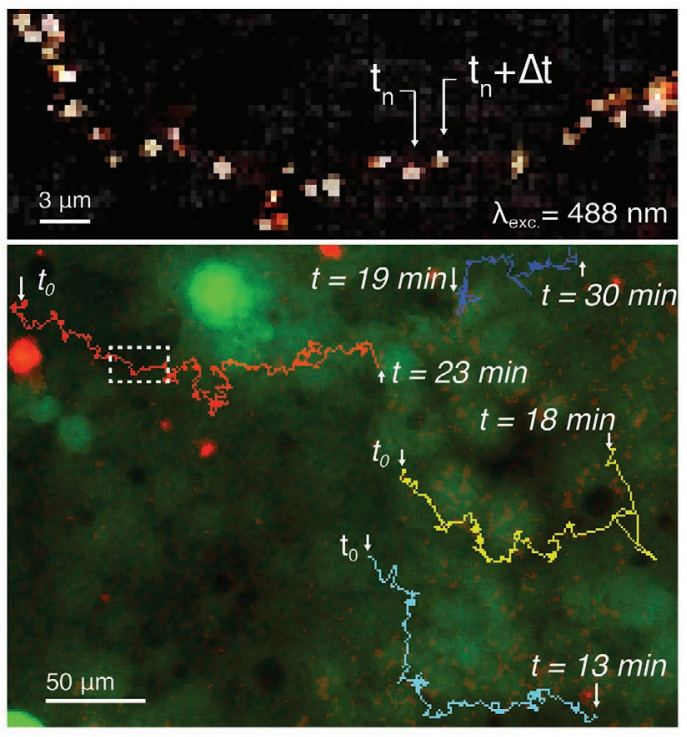

Figure 5. Imaging of Dyes@BNNTs in a HuH6 cells mat. a) Hyperspectral and confocal fluorescence imaging of liver cells incubated with PEG-6T@BNNT for $24 \mathrm{~h}$ and with DiA for 10 min before imaging. The red and green channels correspond to the integrated intensity from 600 to $780 \mathrm{~nm}$ and 490 to $520 \mathrm{~nm}$, respectively. b) Hyperspectral two-photon fluorescence image of fixed hepatoblastoma cells previously incubated for $24 \mathrm{~h}$ with PEG-6T@BNNTs. The dashed line highlights a bleaching test area where the DiA and the PEG-6T@BNNTs were illuminated in twophoton imaging conditions at $\lambda_{\mathrm{ex}}=860 \mathrm{~nm}$ for $30 \mathrm{~min}$. The red and green channels correspond to the integrated intensity from 645 to $715 \mathrm{~nm}$ and from 500 to $530 \mathrm{~nm}$, respectively. c) Fluorescence spectra extracted from the hyperspectral datacube of regions 1 and 2 in (a). d) Two-photon fluorescence excitation profiles of the DiA and PEG-6T@BNNT extracted from an hyperspectral fluorescence image datacube. e) Top: Tracking of a single PEG-6T@BNNT nanoprobe in confluent HuH6 cells using a superposition of luminescence images extracted every 30 s from a time-lapse datacube $\left(\Delta t=650 \mathrm{~ms}, \lambda_{\mathrm{ex}}=488 \mathrm{~nm}\right.$ ). Bottom: Full trajectory reconstruction using a tracking algorithm (see Supporting Information) of the four PEG-6T@BNNTs detected within the field of view.

we reconstructed the trajectory of four individual nanoprobes in the Figure 5e (bottom panel). As expected, the nanoprobes are clearly photostable and the diffusion length is larger than the size of the cells. We do not observe internalization of the nanoprobes in this case, which is probably due to the high coverage of PEGs promoting higher biological furtivity of the nanoprobes.

Previous works on the functionalization of multiwalled BNNTs have shown that BNNTs are outstanding as templates to develop biocompatible nanostructures for drug delivery. ${ }^{[26,63,64]}$ In this work, we have shown that small-diameter BNNTs can template active organic molecules for: 1) unlocking and stabilizing naturally unfavorable aggregation states with dyes that will gain access to complex fluorescence engineering on the nanometer scale for the design of bright emitters; 2) protecting the dyes against photobleaching and chemical degradation mechanisms; 3) reducing the inherent toxicity of organic dyes on living tissues; 4) enabling both chemical processing and easy functionalization of the dyes aggregates, thanks to the BNNT carriers. Capitalizing on these synergetic advances, we have finally highlighted the use of the dyes@BNNTs as fluorescence nanoprobes. Since the preparation of dyes@BNNT is simple and general, we trust that the approach can be further expanded to include other dyes and diameter-sorted BNNTs so as to produce a library of nanoprobes with tuneable colors from the visible down to the NIR I and II for deeper imaging into living organisms.

\section{Experimental Section}

The boron nitride nanotubes (BNNTs) used in this work are provided by BNNT. See Sections S2.1 and S2.2, Supporting Information, for details on cleaning and encapsulation of BNNTs. Briefly, the BNNT powder was sonicated in DMF until complete dispersion, and filtrated on a poly(tetrafluoroethylene) (PTFE) filter $(0.2 \mu \mathrm{m}$ pore size). The obtained film was annealed at $800^{\circ} \mathrm{C}$ for $2 \mathrm{~h}$ under atmospheric conditions. About $5 \mathrm{mg}$ of dyes and $20 \mathrm{mg}$ of purified BNNTs were dispersed sequentially in 20 or $300 \mathrm{~mL}$ of toluene, depending on the dye used, by sonication. The solution was then refluxed at $80^{\circ} \mathrm{C}$ or $115^{\circ} \mathrm{C}$, again depending of the dyes used, to activate the encapsulation. The solution was finally washed in toluene and DMF at least 10 times to remove the free dyes molecules, until the filtrate became colorless. The absorption spectra presented in Figure 1 were acquired using a Bruker vertex v80 spectrometer with $\mathrm{CaF}_{2}$ beamsplitter and $\mathrm{Si}$ and $\mathrm{GaP}$ detector. The fluorescence spectra presented in Figure 1 were recorded using a spectrofluometer from Horiba JY equipped with PMT and InGaAs detectors.

For experiments on individualized Dyes@BNNTs on surface, the Dyes@BNNTs were suspended in N,N-dimethylformamide (DMF) and diluted as needed, typically at a concentration of $\approx 0.1 \mathrm{mg} \mathrm{mL}^{-1}$. This solution was spin-coated at $3000 \mathrm{rpm}$ on a cleaned $\mathrm{Si} / \mathrm{SiO}_{2}$ substrate with aminopropyltriethoxysilane functionalization.

For experiment with Daphnia, an incubation solution was prepared by dispersing Dyes@BNNT in FLAMES culture medium at a concentration ranging from 5 to $100 \mu \mathrm{g} \mathrm{mL}^{-1}$. Adult individuals of $D$. Pulex (clones DISP 1312) were kept at $22^{\circ} \mathrm{C}$ in FLAMES with algae for 4 days before the experiment, allowing for a new reproducing cycle. Then, the specimens were transferred to a FLAMES medium algae-free and remained there for 1 day at minimum to clean the digestive system and limit any PL from algae during the images/time lapse acquisition. Daphniids were then 
placed in the incubation solution for the required time. More details on the experiments with Daphnia and characterizations of the samples are provided in Sections S2.9 and S3, Supporting Information.

The AFM images were produced using a Dimension 3100 microscope. The Raman/PL measurements were taken from different set-up depending on the experiments and these instruments and conditions are summarized in Table S2, Supporting information. In summary, luminescence images and photobleaching experiments were performed at $\lambda_{\mathrm{ex}}=532 \mathrm{~nm}$ using the Raman/PL mapping system RIMA (Photon etc.) using a $100 \times$ objective and a laser power ranging from 0.5 to $80 \mu \mathrm{W} \mu \mathrm{m}^{-2}$. The fluorescence images on Daphnia were acquired with an Olympus confocal microscope equipped with a U-RFL-T mercury light source, a BX-UCB controller and a DP71 digital camera. The images were acquired with a 10X objective and UBG, TRITC and TXRED filters. The acquiring conditions were an exposure time of $1 / 20 \mathrm{~s}$ and ISO 200 sensitivity. Z-stack of 20 images to probe the volume of the Daphnia was acquired by confocal imaging and stacked using the Auto-blend function in Photoshop. The hyperspectral and confocal fluorescence images on confluent $\mathrm{HuH} 6$ hepatoblastoma cells, previously incubated for $24 \mathrm{~h}$ with PEG-6T-BNNT at a concentration ranging from 0.5 to $10 \mu \mathrm{g} \mathrm{mL}$, were recorded on two different setup: a Leica microscope SP8 WLL2 using a HCX Plan Apo CS2 63× oil NA 1.40 objective or a HC PL FLUOTAR 10 $\times / 0.30$ DRY objective and a Leica TCS SP5, using either Argon laser or Mai Tai HP laser (SpectraPhysics, Irvine, USA) and a HC PL APO CS2 $40.0 \times 1.30$ oil objective.

\section{Supporting Information}

Supporting Information is available from the Wiley Online Library or from the author.

\section{Acknowledgements}

This work received financial support from the Natural Sciences and Engineering Research Council of Canada (NSERC) under grants RGPIN2019-06545 and RGPAS-2019-00050 and Canada Research Chairs and the Canada foundation for innovation, 35637. C.A., R.N., P.D., and R.M. acknowledge support from the consortium industriel de recherche et d'innovation en technologies médicales du Québec (MEDTEQ) in collaboration with the partners Photon etc. and La fondation du cancer du sein du Québec. E.G. acknowledges funding from the MarieSkłodowska-Curie-IF 706476-BrightPhoton, the GDRi Graphene and Co, the GDRi multifunctional nanomaterials for travel support, and Graphene Flagship Core 1 (No. 604391). The authors warmly acknowledge G. Wang for support on the Cs-corrected TEM of MPQ - Paris Diderot University and METSA support for access to the Cs-corrected TEM of MPQ. The authors thank Cristescu's team (McGill University) for providing Daphniids, culture medium, algae, and technical assistance, L. Gaboury and J. Hinsinger for access to the Platform of Histology at Institut de Recherche en Immunologie et en Cancerologie (IRIC), and BNNT LLC for providing raw BNNTs. The authors thank the Bordeaux Imaging Center (a service unit of the CNRS-INSERM and Bordeaux University, member of the national infrastructure France Biolmaging supported by the French National Research Agency [ANR-10-INBS-04]) and Sebastien Marais and Christel Poujol for her technical assistance. The authors also thank N. Courtois-Allain and F. Saltel for providing the $\mathrm{HuH} 6$ cell line. G.R. is a board member of the GdR CNRS ImaBio, funded by La Ligue contre le Cancer (Gironde), the Association de Recherche contre le Cancer, and the GSO Cancéropole. Ethical approval for work on Daphnia is not required.

\section{Conflict of Interest}

The authors declare no conflict of interest.

\section{Authors Contributions}

C.A., R.N., F.F., L.S., G.R., A.L., R.M., and E.G. performed the experiments and analyzed the results. C.A., R.M., and E.G. designed the experiments. C.A., R.N., R.G., E.F., and E.G. prepared the samples. P.D., R.M., and E.G. supervised the work and discussed the results. All authors contributed to the scientific discussions, manuscript preparation, and final version.

\section{Keywords}

bio-imaging, nanotube heterostructure, NIR-II fluorescence, molecular aggregation, photostable fluorescence

[1] V. Marx, Nat. Methods 2015, 12, 187

[2] A. Dufton, J. Soc. Dyers Colour. 1894, 10, 90

[3] M. Kasha, Discuss. Faraday Soc. 1950, 9, 14.

[4] J. Widengren, U. Mets, R. Rigler, J. Phys. Chem. 1995, 99, 13368.

[5] R. E. Di Paolo, L. B. Scaffardi, R. Duchowicz, G. M. Bilmes, J. Phys. Chem. 1995, 99, 13796.

[6] W. E. Moerner, M. Orrit, Science 1999, 283, 1670

[7] Al. L. Efros, M. Rosen, Phys. Rev. Lett. 1997, 78, 1110.

[8] C. Galland, Y. Ghosh, A. Steinbrück, M. Sykora, J. A. Hollingsworth, V. I. Klimov, H. Htoon, Nature 2011, 479, 203.

[9] J. Widengren, R. Rigler, Bioimaging 1996, 4, 149.

[10] E. M. S. Stennett, M. A. Ciuba, M. Levitus, Chem. Soc. Rev. 2014, 43, 1057.

[11] A. Diaspro, G. Chirico, C. Usai, P. Ramoino, J. Dobrucki, in Handbook Of Biological Confocal Microscopy (Ed: J. B. Pawley), Springer, Boston, MA, USA 2006, pp. 690-702.

[12] J. White, E. Stelzer, Trends Cell Biol. 1999, 9, 61.

[13] T. Bernas, M. Zarebski, R. R. Cook, J. W. Dobrucki, J. Microsc. 2004, 215, 281.

[14] D. Thomsson, R. Camacho, Y. Tian, D. Yadav, G. Sforazzini, H. L. Anderson, I. G. Scheblykin, Small 2013, 9, 2619.

[15] K. J. Lee, J. H. Oh, Y. Kim, J. Jang, Chem. Mater. 2006, 18, 5002.

[16] M. J. Frampton, H. L. Anderson, Angew. Chem., Int. Ed. 2007, 46, 1028.

[17] G. Yao, L. Wang, Y. Wu, J. Smith, J. Xu, W. Zhao, E. Lee, W. Tan, Anal. Bioanal. Chem. 2006, 385, 518

[18] K. Yanagi, Y. Miyata, H. Kataura, Adv. Mater. 2006, 18, 437.

[19] M. A. Loi, J. Gao, F. Cordella, P. Blondeau, E. Menna, B. Bártová, C. Hébert, S. Lazar, G. A. Botton, M. Milko, C. Ambrosch-Draxl, Adv. Mater. 2010, 22, 1635.

[20] E. Gaufrès, N. Y.-W. Tang, F. Lapointe, J. Cabana, M.-A. Nadon, N. Cottenye, F. Raymond, T. Szkopek, R. Martel, Nat. Photonics 2014, 8, 72.

[21] S. Cambré, J. Campo, C. Beirnaert, C. Verlackt, P. Cool, W. Wenseleers, Nat. Nanotechnol. 2015, 10, 248.

[22] E. Gaufrès, N. Y.-W. Tang, A. Favron, C. Allard, F. Lapointe, V. Jourdain, S. Tahir, C.-N. Brosseau, R. Leonelli, R. Martel, ACS Nano 2016, 10, 10220.

[23] S. van Bezouw, D. H. Arias, R. Ihly, S. Cambré, A. J. Ferguson, J. Campo, J. C. Johnson, J. Defillet, W. Wenseleers, J. L. Blackburn, ACS Nano 2018, 12, 6881.

[24] X. Blase, A. Rubio, S. G. Louie, M. L. Cohen, Europhys. Lett. 1994, 28, 335.

[25] R. Arenal, O. Stéphan, M. Kociak, D. Taverna, A. Loiseau, C. Colliex, Phys. Rev. Lett. 2005, 95, 127601. 
[26] J. Niskanen, I. Zhang, Y. Xue, D. Golberg, D. Maysinger, F. M. Winnik, Nanomedicine 2016, 11, 447.

[27] A. Merlo, V. R. S. S. Mokkapati, S. Pandit, I. Mijakovic, Biomater. Sci. 2018, 6, 2298.

[28] H. Chen, Y. Chen, J. Yu, J. S. Williams, Chem. Phys. Lett. 2006, 425, 315.

[29] N. Chejanovsky, Y. Kim, A. Zappe, B. Stuhlhofer, T. Taniguchi, K. Watanabe, D. Dasari, A. Finkler, J. H. Smet, J. Wrachtrup, Sci. Rep. 2017, 7, 14758.

[30] F. C. Spano, J. Clark, C. Silva, R. H. Friend, J. Chem. Phys. 2009, 130, 074904.

[31] D. P. Ostrowski, L. A. Lytwak, M. L. Mejia, K. J. Stevenson, B. J. Holliday, D. A. Vanden Bout, ACS Nano 2012, 6, 5507.

[32] A. J. Musser, S. K. Rajendran, K. Georgiou, L. Gai, R. T. Grant, Z. Shen, M. Cavazzini, A. Ruseckas, G. A. Turnbull, I. D. W. Samuel, J. Clark, D. G. Lidzey, J. Mater. Chem. C 2017, 5, 8380.

[33] Y. Almadori, L. Alvarez, R. Le Parc, R. Aznar, F. Fossard, A. Loiseau, B. Jousselme, S. Campidelli, P. Hermet, A. Belhboub, A. Rahmani, T. Saito, J.-L. Bantignies, J. Phys. Chem. C 2014, 118, 19462.

[34] A. Belhboub, P. Hermet, L. Alvarez, R. Le Parc, S. Rols, A. C. Lopes Selvati, B. Jousselme, Y. Sato, K. Suenaga, A. Rahmani, J.-L. Bantignies, J. Phys. Chem. C 2016, 120, 28802.

[35] S. T. Skowron, T. W. Chamberlain, J. Biskupek, U. Kaiser, E. Besley, A. N. Khlobystov, Acc. Chem. Res. 2017, 50, 1797.

[36] E. Jelley, 1936, 138, 1009.

[37] F. C. Spano, Annu. Rev. Phys. Chem. 2006, 57, 217.

[38] F. C. Spano, C. Silva, Annu. Rev. Phys. Chem. 2014, 65, 477.

[39] H. Manzano, I. Esnal, T. Marqués-Matesanz, J. Bañuelos, I. López-Arbeloa, M. J. Ortiz, L. Cerdán, A. Costela, I. García-Moreno, J. L. Chiara, Adv. Funct. Mater. 2016, 26, 2756.

[40] C. Sun, B. Li, M. Zhao, S. Wang, Z. Lei, L. Lu, H. Zhang, L. Feng, C. Dou, D. Yin, H. Xu, Y. Cheng, F. Zhang, J. Am. Chem. Soc. 2019, 141, 19221.

[41] T. Tanaka, M. Ishitobi, T. Aoyama, S. Matsumoto, Langmuir 2016 , 32, 4710

[42] M. Grabolle, M. Spieles, V. Lesnyak, N. Gaponik, A. Eychmüller, U. Resch-Genger, Anal. Chem. 2009, 81, 6285.

[43] U. Resch-Genger, M. Grabolle, S. Cavaliere-Jaricot, R. Nitschke, T. Nann, Nat. Methods 2008, 5, 763.

[44] Y. Kanemitsu, N. Shimizu, K. Suzuki, Y. Shiraishi, M. Kuroda, Phys. Rev. B 1996, 54, 2198.

[45] E. Da Como, M. A. Loi, M. Murgia, R. Zamboni, M. Muccini, J. Am. Chem. Soc. 2006, 128, 4277

[46] E. Gaufrès, S. Marcet, V. Aymong, N. Y.-W. Tang, A. Favron, F. Thouin, C. Allard, D. Rioux, N. Cottenye, M. Verhaegen, R. Martel, J. Raman Spectrosc. 2018, 49, 174.

[47] A. Siciliano, R. Gesuele, G. Pagano, M. Guida, J. Biotechnol. Biodiversity 2015, 1, 1.

[48] M. P. Celis-Salgado, A. Cairns, N. Kim, N. D. Yan, Verh. Int. Ver. Limnol. 2008, 30, 265.

[49] J. W. Churchman, J. Am. Med. Assoc. 1925, 85, 1849.
[50] K. Welsher, Z. Liu, S. P. Sherlock, J. T. Robinson, Z. Chen, D. Daranciang, H. Dai, Nat. Nanotechnol. 2009, 4, 773.

[51] K. Welsher, S. P. Sherlock, H. Dai, Proc. Natl. Acad. Sci. USA 2011, 108, 8943.

[52] N. Danné, A. G. Godin, Z. Gao, J. A. Varela, L. Groc, B. Lounis, L. Cognet, ACS Photonics 2018, 5, 359.

[53] D. Roxbury, P. V. Jena, R. M. Williams, B. Enyedi, P. Niethammer, S. Marcet, M. Verhaegen, S. Blais-Ouellette, D. A. Heller, Sci. Rep. 2015, 5, 14167.

[54] L. Shi, L. A. Sordillo, A. Rodríguez-Contreras, R. Alfano, J. Biophotonics 2016, 9, 38.

[55] A. L. Antaris, H. Chen, K. Cheng, Y. Sun, G. Hong, C. Qu, S. Diao, Z. Deng, X. Hu, B. Zhang, X. Zhang, O. K. Yaghi, Z. R. Alamparambil, X. Hong, Z. Cheng, H. Dai, Nat. Mater. 2016, 15, 235.

[56] M. Waldherr, M. Mišík, F. Ferk, J. Tomc, B. Žegura, M. Filipič, W. Mikulits, S. Mai, O. Haas, W. W. Huber, E. Haslinger, S. Knasmüller, Arch. Toxicol. 2018, 92, 921.

[57] P. Godoy, N. J. Hewitt, U. Albrecht, M. E. Andersen, N. Ansari, S. Bhattacharya, J. G. Bode, J. Bolleyn, C. Borner, J. Böttger, A. Braeuning, R. A. Budinsky, B. Burkhardt, N. R. Cameron, G. Camussi, C.-S. Cho, Y.-J. Choi, J. C. Rowlands, U. Dahmen, G. Damm, O. Dirsch, M. T. Donato, J. Dong, S. Dooley, D. Drasdo, R. Eakins, K. S. Ferreira, V. Fonsato, J. Fraczek, R. Gebhardt, A. Gibson, M. Glanemann, C. E. P. Goldring, M. J. Gómez-Lechón, G. M. M. Groothuis, L. Gustavsson, C. Guyot, D. Hallifax, S. Hammad, A. Hayward, D. Häussinger, C. Hellerbrand, P. Hewitt, S. Hoehme, H.-G. Holzhütter, J. B. Houston, J. Hrach, K. Ito, H. Jaeschke, V. Keitel, J. M. Kelm, B. Kevin Park, C. Kordes, G. A. Kullak-Ublick, E. L. LeCluyse, P. Lu, J. Luebke-Wheeler, A. Lutz, D. J. Maltman, M. Matz-Soja, P. McMullen, I. Merfort, S. Messner, C. Meyer, J. Mwinyi, D. J. Naisbitt, A. K. Nussler, P. Olinga, F. Pampaloni, J. Pi, L. Pluta, S. A. Przyborski, A. Ramachandran, V. Rogiers, C. Rowe, C. Schelcher, K. Schmich, M. Schwarz, B. Singh, E. H. K. Stelzer, B. Stieger, R. Stöber, Y. Sugiyama, C. Tetta, W. E. Thasler, T. Vanhaecke, M. Vinken, T. S. Weiss, A. Widera, C. G. Woods, J. J. Xu, K. M. Yarborough, J. G. Hengstler, Arch. Toxicol. 2013, 87, 1315.

[58] E. S. Ruthazer, H. T. Cline, Real-Time Imaging 2002, 8, 175.

[59] M. Lan, S. Zhao, Y. Xie, J. Zhao, L. Guo, G. Niu, Y. Li, H. Sun, H. Zhang, W. Liu, J. Zhang, P. Wang, W. Zhang, ACS Appl. Mater. Interfaces 2017, 9, 14590.

[60] A. G. Godin, J. A. Varela, Z. Gao, N. Danné, J. P. Dupuis, B. Lounis, L. Groc, L. Cognet, Nat. Nanotechnol. 2017, 12, 238.

[61] N. Fakhri, F. C. MacKintosh, B. Lounis, L. Cognet, M. Pasquali, Science 2010, 330, 1804.

[62] J.-Y. Tinevez, N. Perry, J. Schindelin, G. M. Hoopes, G. D. Reynolds, E. Laplantine, S. Y. Bednarek, S. L. Shorte, K. W. Eliceiri, Methods 2017, 115, 80.

[63] S. Velayudham, C. H. Lee, M. Xie, D. Blair, N. Bauman, Y. K. Yap, S. A. Green, H. Liu, ACS Appl. Mater. Interfaces 2010, 2, 104.

[64] G. Ciofani, G. G. Genchi, I. Liakos, A. Athanassiou, D. Dinucci, F. Chiellini, V. Mattoli, J. Colloid Interface Sci. 2012, 374, 308. 